\title{
La Kerlan Collection à l'Université du Minnesota
}

\section{Lisa Von Drasek}

\section{OpenEdition}

\section{Journals}

\section{Édition électronique}

URL : http://journals.openedition.org/genesis/4179

DOI : 10.4000/genesis.4179

ISSN : 2268-1590

\section{Éditeur :}

Presses universitaires de Paris Sorbonne (PUPS), Société internationale de génétique artistique littéraire et scientifique (SIGALES)

\section{Édition imprimée}

Date de publication : 1 juin 2019

Pagination : 147-148

ISSN : 1167-5101

\section{Référence électronique}

Lisa Von Drasek, "La Kerlan Collection à I'Université du Minnesota », Genesis [En ligne], 48| 2019, mis en ligne le 01 juin 2020, consulté le 25 janvier 2021. URL : http://journals.openedition.org/genesis/ 4179 ; DOI : https://doi.org/10.4000/genesis.4179 


\title{
La Kerlan Collection à l'université du Minnesota
}

\author{
Lisa Von Drasek
}

$\mathrm{L}$ e département des archives et des collections spéciales (ASC : Archives and Special Collections) des bibliothèques de l'université du Minnesota abrite plusieurs fonds de recherche sur la littérature pour enfants, rassemblés au sein du CLRC (Children's Literature Research Collections ${ }^{1}$ ). Il s' agit d'une bibliothèque et d'un fonds internationalement reconnus sur la littérature pour la jeunesse, couvrant les périodes moderne et contemporaine, réunissant des livres, des manuscrits, des illustrations, des bandes dessinées, des publications périodiques telles que les «story papers » et d'autres documents relatifs à la création d'œuvres pour enfants, y compris des documents d'archives et des planches originales de plus de 1700 auteurs et illustrateurs.

Au sein de ces archives, la Kerlan Collection, qui célébrera ses 70 ans d'existence en 2019, tient une place particulière. Fondée par le Dr Irvin Kerlan (1912-1963), elle réunit plus de 100000 livres pour enfants ainsi que des manuscrits originaux, des œuvres d'art, des placards et des épreuves en couleurs ainsi que d'autres documents génétiques. Le Dr Kerlan commença par acheter chaque année les œuvres de littérature pour la jeunesse les plus réussies et les plus représentatives et, lorsqu'il pouvait se le permettre, les classiques de la littérature pour enfants et les œuvres ayant obtenu le prix Newbery par le passé. Il chercha bientôt à aller plus loin en faisant l'acquisition de tous les documents qui témoignent de la genèse des livres. Il écrivit aux auteurs et aux illustrateurs qui lui répondirent en lui envoyant leurs manuscrits originaux, leurs dessins et leurs correspondances avec leurs éditeurs et leurs jeunes lecteurs. Le fonds Kerlan continue à se développer grâce aux généreuses donations des écrivains et des artistes qui comprennent l'importance de la CLRC, véritable «sanctuaire» pour le travail de toute une vie.

Le fonds Kerlan mène une politique active d'acquisition et de conservation de ces matériaux et les met à disposition des chercheurs. Il parraine également des conférences et des journées d'études. Il monte des expositions dans ses

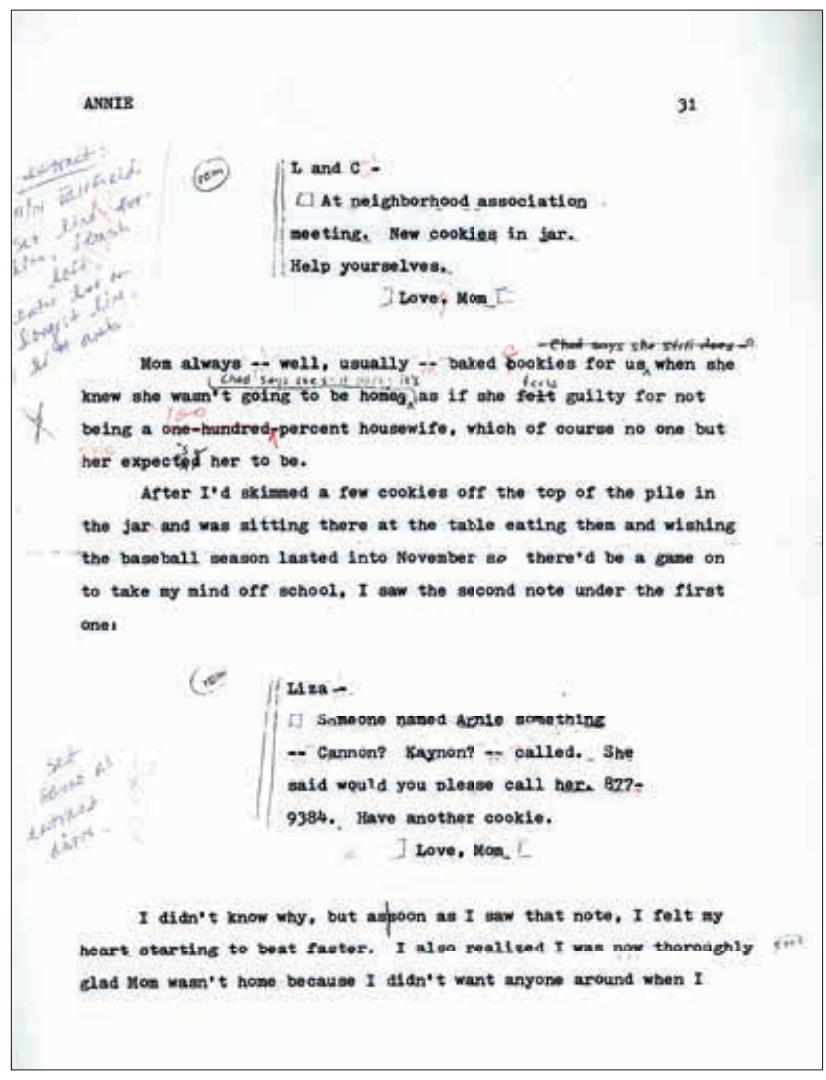

Fig. 1 : Nancy Garden, tapuscrit révisé du roman Annie on my mind (1982).

Reproduit avec l'aimable autorisation de l'université du Minnesota. CLibraries Archives and Special Collections, Kerlan Collection

lieux et prête des documents pour des expositions au niveau national et international. En janvier 2019, l'exposition «The ABC of It: Why Children's Books Matter» explorera l'impact social et culturel de la littérature pour la jeunesse

1. < https://www.lib.umn.edu/clrc >. 


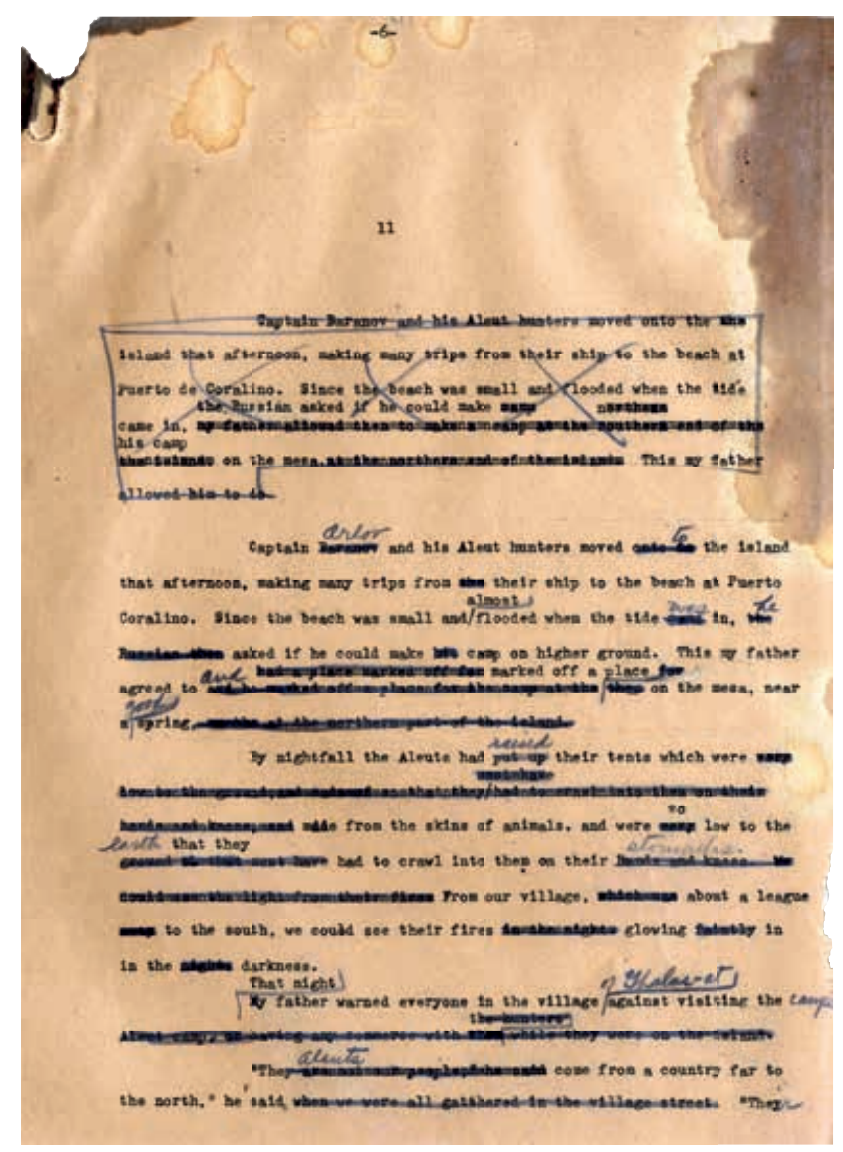

Fig. 2 : Scott O’Dell, tapuscrit révisé du roman Island of the Blue Dolphins (1960).

Reproduit avec l'aimable autorisation de l'Université du Minnesota. (CLibraries Archives and Special Collections, Kerlan Collection

à travers l'histoire en s'appuyant sur le cadre théorique de l'historien des livres pour enfants Leonard Marcus et sur ses descriptions éclairées (et parfois ironiques), en l'illustrant de plus de deux cents pièces incluant des œuvres d'art originales, des lettres, des livres et des documents issus du fonds Kerlan et d'autres collections spécialisées. Plus récemment, les illustrations de Virginia Lee Burton pour son album The Little House (1942) ont fait l'objet d'une exposition à Tokyo. En tant que conservatrice, je donne régulièrement des conférences sur le processus de révision, sur la genèse des albums et sur les relations entre les éditeurs et les auteurs dont témoignent les documents de travail, textuels et iconographiques, présents dans les collections.

En raison de la nature exceptionnelle de notre fonds, les documents conservés dans notre département doivent habituellement être consultés sur place. Cependant, des expositions en ligne nous permettent de mettre les ressources et l'expertise de la CLRC à la disposition des chercheurs, des étudiants et des enseignants du monde entier. Parmi ces expositions en ligne, on trouve «Le Petit Chaperon Rouge : étude comparative d'un conte populaire», «Illustrer des livres pour enfants : techniques et média», «La fabrique des illustrations des albums : qu'est-ce que la quadrichromie?», «Balloons Over Broadway, Melissa Sweet et la conception d'un livre pour enfants $\gg 2$.

Nous tenons notre communauté d'amateurs de livres pour enfants et pour jeunes adultes informée de nos acquisitions, expositions et évènements dans notre lettre d'information The Blue Ox Review ${ }^{3}$.

Traduit de l'anglais par Christine Collière-Whiteside

2. «Little Red Riding Hood: A Comparative Folk Tale Study», «Children's Book Art: Techniques and Media», «The Making of Picture Book Illustrations: What is Preseparated Art?», «Balloons Over Broadway, Melissa Sweet, and the Engineering of a Children's Book». Ces expositions peuvent être visitées à l'adresse suivante : https://www.lib.umn.edu/ clrc/digital-resources

3. https://www.continuum.umn.edu/kerlan/ 